\title{
Colour Features Extraction Techniques and Approaches for Content-Based Image Retrieval (CBIR) System
}

\author{
Muhammad Naim Abdullah ${ }^{1,2}{ }^{*}$, Mohd Afizi Mohd Shukran', Mohd Rizal Mohd Isa1, \\ Nor Suraya Mariam Ahmad1, Mohammad Adib Khairuddin', Mohd Sidek Fadhil Mohd Yunus ${ }^{3}$, \\ Fatimah Ahmad 1
}

\author{
${ }^{1}$ Universiti Pertahanan Nasional Malaysia (UPNM), Kuala Lumpur, Malaysia \\ ${ }^{2}$ University Malaysia of Computer Science and Engineering (UNIMY), Selangor, Malaysia \\ ${ }^{3}$ Universiti Pendidikan Sultan Idris (UPSI), Perak, Malaysia \\ Email: *m.naim.abdullah@gmail.com
}

How to cite this paper: Abdullah, M.N., Shukran, M.A.M., Isa, M.R.M., Ahmad, N.S.M., Khairuddin, M.A., Yunus, M.S.F.M. and Ahmad, F. (2021) Colour Features Extraction Techniques and Approaches for Content-Based Image Retrieval (CBIR) System. Journal of Materials Science and Chemical Engineering, 9, 29-34. https://doi.org/10.4236/msce.2021.97003

Received: July 2, 2021

Accepted: July 25, 2021

Published: July 28, 2021

\begin{abstract}
An image retrieval system was developed purposely to provide an efficient tool for a set of images from a collection of images in the large database that matches the user's requirements in similarity evaluations such as image content similarity, edge, and colour similarity. Retrieving images based on the contents which are colour, texture, and shape is called content-based image retrieval (CBIR). This paper discusses and describes about the colour features technique for image retrieval systems. Several colour features technique and algorithms produced by the previous researcher are used to calculate the similarity between extracted features. This paper also describes about the specific technique about the colour basis features and combined features (hybrid techniques) between colour and shape features.
\end{abstract}

\section{Keywords}

Content-Based Image Retrieval, Colour Features, CBIR

\section{Introduction}

In current technological information trend, the internet has dramatically revolutionized many different fields and become widely available. Due to rapid growth of internet, all image information and data are collected and digitized. Every internet user is able to access them because all the image information and data are available in the digital image format. An efficient technique and method are needed to access this huge amount of data for querying the indexed image data- 
base. In order to find and locate the needed information from these large databases, it is difficult and surely will take a lot of time. So, a method called content-based image retrieval is the efficient retrieval method to find the relevant and needed images. Content-based Image Retrieval (CBIR) system was introduced in 1990 [1]. It is basically a technique used for automatic retrieval of images in a large database that perfectly matches the query image.

Generally, a basic CBIR system architecture consists of six functional blocks which is image database (the block that contains image of the chosen database), feature database (can be characterised by a set of features), feature extraction (features may be text-based features and visual features), query image (can be any of the database images), image matching and indexing (the features of the query image are compared with features that are already stored in the feature database), and retrieved image (the system that will select any number of images having the highest overall similarity to the given query image and present to the user as retrieved images) [2]. Figure 1 shows the typical architecture of a CBIR system whereas Figure 2 shows the typical architecture of a Colour CBIR system.

\section{CBIR General Techniques}

Basically, a CBIR system aims at retrieving relevant images from the image database on the basis of automatically derived features of the image. In CBIR systems, while building an image database or retrieving an image from the database, feature vectors are the first extracted from images. The features can be colour, texture, shape, and the combination of these features (hybrid features) [2]. These features are then used to build the feature database. When a query image is given, its feature vectors are extracted, and similarity matching is performed with the feature vectors already present in the feature database as illustrated in Figure 1 in the previous section.

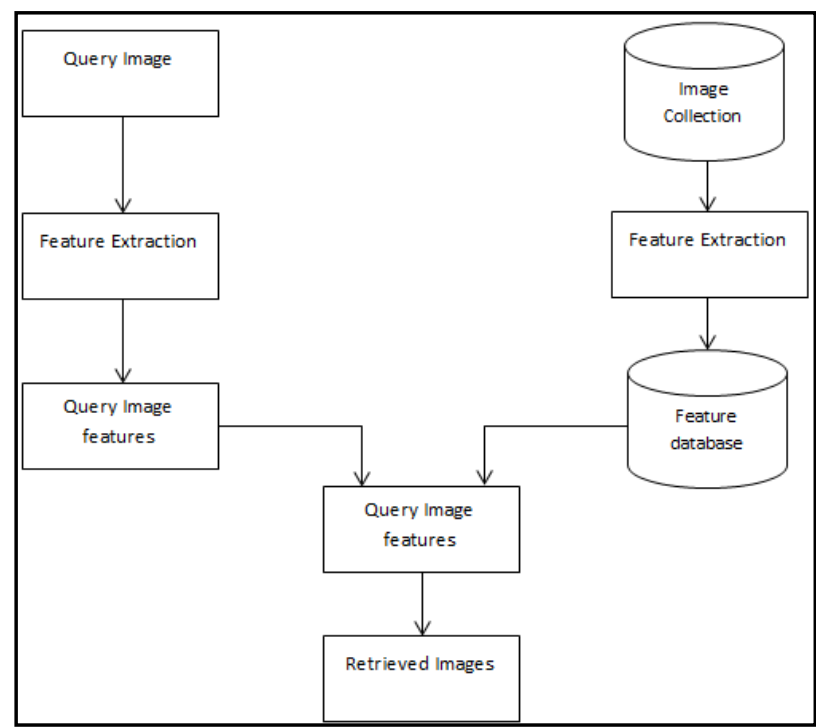

Figure 1. Typical architecture of CBIR system. 


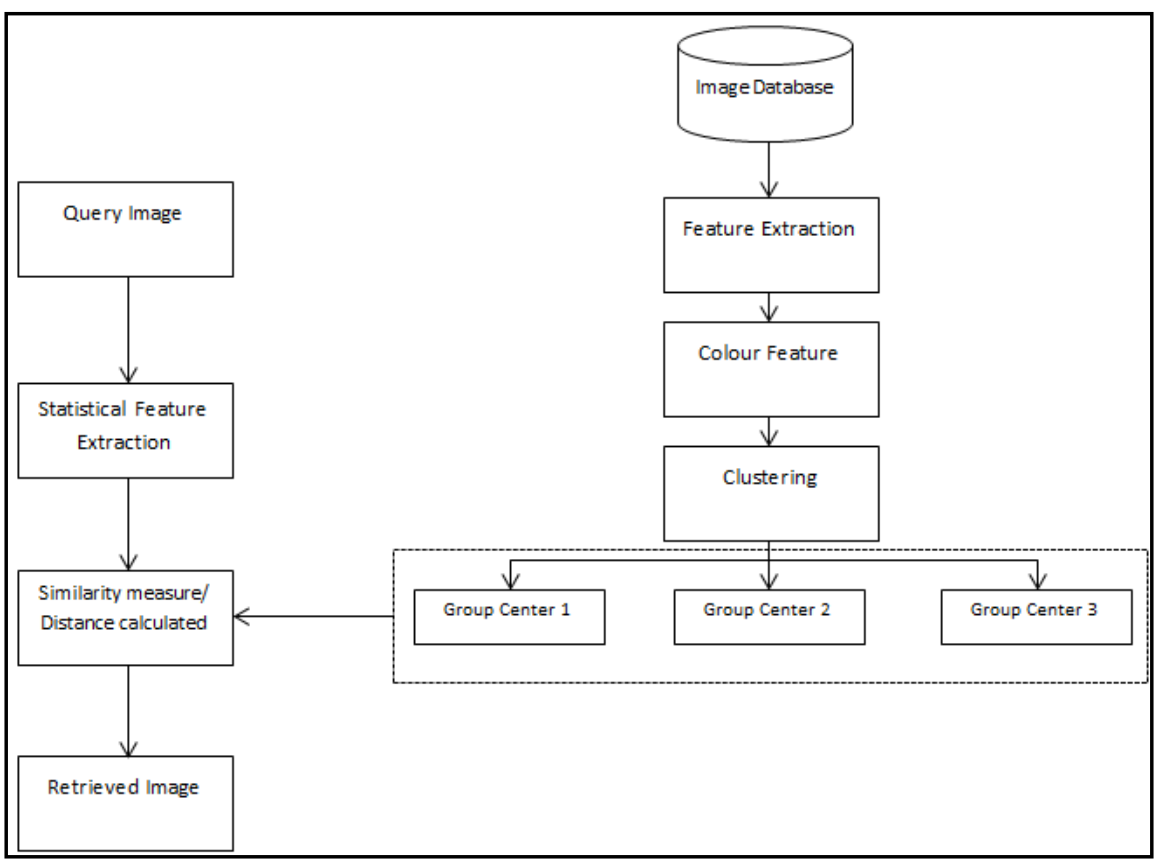

Figure 2. Colour content-based image retrieval system architecture.

The techniques of CBIR to be used depend on the image database. Images are indexed by their visual content such as colour, texture, shape, and their combination in CBIR. The first technique of CBIR to be discussed in this section is colour features. Colour is one of the important features that make possible the recognition of image by human. In [3] state that the colour is one of the most widely used low-level visual features and is invariant to image size and orientation. According to [4], colour features are the most expressive of all the visual features and have been extensively used in the image retrieval system. Other than that, in CBIR system, another important visual feature is shape. According to [5], shape is one of the basic features used to describe image content and it can also be used to provide powerful information. Nevertheless, shape representation and description is a difficult task because when a 3-D real world object is projected onto a 2-D image plane, one dimension of object information is lost. As a result, the shape extracted from the image only partially represents the projected object. Further, shape is often corrupted with noise, defects, arbitrary distortion and occlusion.

Another important element in visual perception is "Texture". Texture also can be used to separate regions of interest in an image. According to [6], texture is also one of the most used low level visual features that refer to innate surface properties of an object and their relationship to the surrounding environment and it also contains important information about the structural arrangement of surfaces and their relationship with the surroundings.

\section{CBIR Techniques Using Colour Features}

Colour is the most effective of all the visual features and has been broadly used 
in the image retrieval systems [4]. Colours are usually defined in 3-dimensional colour areas. These may either be Red, Green, and Blue (RGB), Hue, Saturation, and Value (HSV) or Hue, Saturation, and Brightness (HSB). The last two which is Hue, Saturation, and Value (HSV) and Hue, Saturation, and Brightness (HSB) are based on the human perception of hue, saturation, and brightness [7].

Most image formats like Joint Photographic Experts Group (JPEG), Bitmap (BMP), Graphics Interchange Format (GIF), use the RGB colour area to store image information. The RGB colour area is outlined as a unit cube with red, green, and blue axes. Thus, a vector with 3 co-ordinates represents the colour for this image area. Once all 3 coordinates are set to zero the colour perceived is black. Once all 3 coordinates are set to one, the colour perceived is white [8]. The other colour areas operate in an exceedingly similar fashion however with a special perception.

\subsection{RGB Colour Histogram}

In 2012, previous researcher has proposed a colour matching method based on colour indexing also known as colour histogram intersection method and it is based on matching of colour histograms in early 1990s [2]. RGB colour histogram has been selected to determine the features of the colour and the mean, standard deviation, normalized relative smoothness, third moment, forth moment, uniformity and entropy is used to calculate the texture feature. Combining the texture and colour features will produce the better result in CBIR [9].

\subsection{RGB Colour Space}

RGB colour space has been used for feature extraction of query image and database image [10]. On the other features which are extracted features, [10] used hierarchical clustering algorithm for initial clusters formation afterward thereon use the clusters K-Means clustering algorithm. Hierarchical clustering helps for quicker image retrieval and improves the searching process by providing correct direction for search. K-Means clustering algorithm provides the correct result of image from an outsized database. Due to use of two clustering techniques, complexity gets increased [11].

\subsection{RGB Conversion}

RGB image has been converted and applied into grayscale image and it is followed by calculating the texture primitive of that grayscaled image by dividing it into different block sizes [12]. This method improves and it also gives better result than the combination of texture and colour feature extraction [9].

\subsection{Discrete Cosine Transform (DCT)}

Colour features have been widely used and calculated for CBIR. [13] use Discrete Cosine Transform (DCT) to gain compaction of the image energy. In order to create image clusters, $\mathrm{K}$-means is used. At the same time, to match and retrieve 
the images, chi-square distance measure is used [13].

\subsection{Hybrid of Colour and Shape Features}

Colour and shape features integration will give better result than using single feature extraction technique [14]. With trademark image, in terms of retrieval accuracy, it shows that colour basis is more accurate than the shape-based image. By integrating both (colour and shape) features, it will increase the speed of retrieval process by using a branch and bound method to compute the closest neighbors for the query image without affecting the strength or robustness of the system. Retrieving results become more efficient and effective by combine and integrating the easily attracted features [14].

\section{Conclusions}

Image features play an important role or function in retrieving accurate and suitable images from the large image database. Effective image retrieval from database requires exploration of suitable features such as colour, text and shape of images in the database. Appropriately, many previous researchers have presented and proposed several image retrieval techniques and algorithms by exploring various features and trans-forms.

In this paper, CBIR techniques and algorithms by using colour features are briefly described and discussed. Based on the related study, a colour matching method based on colour indexing or colour histogram has been introduced in the early 1990s. Besides that, RGB colour space technique also has been used for feature extraction of query image and database image.

Other than that, RGB conversion is also one of the successful techniques by converting and applying the RGB image into grayscale image and it is followed by calculating the texture primitive of that grayscale image by dividing it into different block sizes. Previous researcher also invents and uses the Discrete Cosine Transform (DCT) technique to gain compaction of the image energy and use the K-Means in order to create image clusters. Last but not least, combining and integrating the colour and shape features will produce better image retrieval result than using single feature extraction technique.

\section{Acknowledgements}

This research work is supported by Universiti Pertahanan Nasional Malaysia (UPNM), and University Malaysia of Computer Science and Engineering (UNIMY). This research also was done in conjunction with the main research titled Research on Content-Based Image Retrieval (CBIR) Approach for Biometric Security Using RGB Histogram for Colour Features Extraction.

\section{Conflicts of Interest}

The authors declare no conflicts of interest regarding the publication of this paper. 


\section{References}

[1] Shriwas, M.K. and Raut, V.R. (2015) Content Based Image Retrieval: A Past, Present and New Feature Descriptor. 2015 International Conference on Circuits, Power and Computing Technologies [ICCPCT-2015], 1-7. https://doi.org/10.1109/ICCPCT.2015.7159404

[2] Rao, C.S. and Kumar, S.S. (2012) Content-Based Image Retrieval Fundamentals \& Algorithms. LAP Lambert Academic Publishing.

[3] Yue, J., Li, Z., Liu, L. and Fu, Z. (2011) Content-Based Image Retrieval Using Color and Texture Fused Features. Mathematical and Computer Modelling, 54, 1121-1127. https://doi.org/10.1016/J.MCM.2010.11.044

[4] Manjunath, B.S., Ohm, J.R., Vasudevan, V.V. and Yamada, A. (2001) Color and Texture Descriptors. IEEE Transactions on Circuits and Systems for Video Technology, 11, 703-715. https://doi.org/10.1109/76.927424

[5] Zhang, D. and Lu, G. (2004) Review of Shape Representation and Description Techniques. Pattern Recognition, 37, 1-19. In Pattern Recognition (Vol. 37). https://doi.org/10.1016/j.patcog.2003.07.008

[6] Gandhani, S. and Singhal, N. (2016) Content Based Image Retrieval: Survey and Comparison of CBIR System Based on Combined Features. International Journal of Signal Processing, Image Processing and Pattern Recognition, 8, 417-422. https://doi.org/10.14257/ijsip.2015.8.11.37

[7] Toselli, A.H., Vidal, E., Casacuberta, F., Toselli, A.H., Vidal, E. and Casacuberta, F. (2011) Active Learning Methods for Interactive Image Retrieval. Multimodal Interactive Pattern Recognition and Applications, 17, 209-226. https://doi.org/10.1007/978-0-85729-479-1 11

[8] Suhasini, P.S., Krishna, K. and Krishna, I.V. (2009) CBIR Using Color Histogram Processing. Journal of Theoretical and Applied Information Technology.

[9] Thawari, P.B. and Janwe, N.J. (2011) CBIR Based on Color and Texture. International Journal of Information Technology and Knowledge Management, 4, 129-132.

[10] Murthy, V.S.V.S., Vamsidhar, E., Kumar, J.N.V.R.S. and Sankara Rao, P. (2012) Content Based Image Retrieval Using Hierarchical and K-Means Clustering Techniques. International Journal of Engineering Science and Technology, 2, 209-212.

[11] Shukran, M.A.M., Abdullah, M.N. and Yunus, M.S.F.M. (2021) New Approach on the Techniques of Content-Based Image Retrieval (CBIR) Using Color, Texture and Shape Features. Journal of Materials Science and Chemical Engineering, 9, 51-57. https://doi.org/10.4236/msce.2021.91005

[12] Fazal-e-Malik and Baharudin, B. (2011) Efficient Image Retrieval Based on Texture Features. 2011 National Postgraduate Conference, 1-6. https://doi.org/10.1109/NatPC.2011.6136308

[13] Talbar, S.N. and Varma, S.L. (2009) iMATCH: Image Matching and Retrieval for Digital Image Libraries. 2009 Second International Conference on Emerging Trends in Engineering \& Technology, 196-201. https://doi.org/10.1109/ICETET.2009.119

[14] Jain, A.K. and Vailaya, A. (1995) Image Retrieval Using Color and Shape 1 Introduction. Image. Rochester, New York. 